\title{
Distribution of Oscillator Strengths for Recombination of Localised Excitons in Two Dimensions
}

\author{
MICHAEL WHLKINSON, PAUL. N. WALKER and KAUMBA CHINYAMA \\ Department of Physics and Applied Physics, John Anderson Building, University of Strathclyde, Glasgow G4 0NG. \\ Scotland, UK
}

\begin{abstract}
We investigate the distribution of oscillator strengths for the recombination of excitons in a two-dimensional sample, trapped in local minima of the confinement potential: the results are derived from a statistical topographic model of the potential. The predicted distribution of oscillator strengths is very different from the Porter-Thomas distribution which usually characterises disordcred systems, and is notable for the fact that small oscillator strengths are extremely rare. (C) 1997 Elsevier Science Ltd
\end{abstract}

\section{INTRODUCTION}

Many experiments have been performed in which excitons (excitations in the form of an electron-hole bound state) are trapped in a layer of lower bandgap semiconductor, between two layers of higher bandgap material $[1,2]$. Exciton absorption and emission lines in these two-dimensional samples are typically much broader than in three-dimensional systems. This is often due to fluctuations in the width of the layer: there is a 'quantum confinement' contribution to the energy of the exciton, which is a decreasing function $E(w)$ of the layer width $w$ and which is analogous to the ground state energy $\pi^{2} \hbar^{2} / 2 m w^{2}$ of a particle trapped in a one-dimensional potential well $[3,4]$.

The excitons are able to interact with phonons by radiationless processes $[5,6]$, becoming trapped in local minima of the confinement energy: the lifetime for electron-hole recombination is typically long enough that most of the luminescence observed from these samples is from trapped excitons. This trapping effect has recently been observed directly by spatially resolved studies of exciton luminescence $[7,8]$ : if the luminescence is recorded from a macroscopic area of the sample, a broad spectrum is observed, whereas sufficiently small microscopic areas show either no luminescence or a small number of relatively sharp lines, corresponding to excitons in a single trap. These exciton traps may be thought of as a novel type of mesoscopic system.

The spectra of two-dimensional excitons show some near-universal features; for example, it was noticed that the Stokes shift $S$ of the luminescence spectrum relative to the absorption peak and the width $W$ of the absorption spectrum usually satisfy $S / W \approx 0.6$, independent of the semiconductor materials or of the magnitude of the broadening [9]. This observation was explained using a classical picture of trapping of excitons in local minima of a smooth effective confinement potential, modelled as a Gaussian random function [10]. This statistical topographic model predicted $S / W=0.55 \ldots$, in good agreement with the experimental values. In Section 2, we review this model and discuss a variant of the experimental approach which may give better agreement with theoretical predictions. 
In this paper, we discuss the distribution of oscillator strengths (or, equivalently, Einstein A coefficients) for exciton recombination, for excitons localised in minima of the effective confinement potential. We use the same statistical topographic model as was previously used to calculate the Stokes shift. If the model is correct, the probability distribution of oscillator strengths for localised excitons should be a near universal signature of trapped excitons, but we believe that this may be a more sensitive test of the validity of the model. The predicted probability distribution of oscillator strengths is

$$
P(I)=I^{-9} \exp \left(\frac{1}{2 I^{4}}\right) \operatorname{erfc}\left(\frac{\sqrt{3}}{2 I^{2}}\right)
$$

(The scaling of the intensities $I$ is arbitrary; for this form of the distribution, the mean intensity is $\langle I\rangle \approx 0.699 \ldots$...) This result is interesting because the predicted distribution has very few small intensities: the fraction of recombination lines predicted to be less than half the mean intensity is approximately $3.15 \times 10^{-7}$. It is very different from the Porter-Thomas distribution $P(I) \sim \exp (-I) / \sqrt{I}$, which usually characterises the distribution of oscillator strengths for disordered or complex systems [11], and which follows from the Gaussian distribution of matrix elements in such systems.

Section 3 discusses the model used for calculating the matrix elements for recombination of excitons trapped in local mimima of a potential. In Section 4 , we give a complete characterisation of the distribution of quadratic forms characterising the stationary points of an isotropic Gaussian random function: although this is not difficult to obtain using standard techniques of statistical topography $[12,13]$, we could not find a discussion of it in the literature in the form we require. These results are then used to obtain equation (1).

The field of statistical topography was largely stimulated by the desire to understand optical properties of random surfaces, such as that of the sea. In Section 4, we also comment on the closest optical analogy of our results, namely the distribution of intensities of reflected images of a small light source on a distant random surface, (such as 'sea glitter', reflections of the sun from the sea, observed from an aircraft [14]). The distribution of oscillator strengths turns out to be very different from that of the intensities of sea glitter sparkles.

\section{THE STATISTICAL TOPOGRAPHIC MODEL}

Here we briefly review the statistical topographic model for exciton luminescence presented in Refs $[9,10]$. We also propose a reason for the small discrepancy between theory and experiment, and a variant of the experimental approach which may give a better agrecment with experiment.

Figure 1 shows the absorption and luminescence spectra of excitons in a semiconductor heterostructure: the data are taken from Ref. [15]. First, consider the form of the absorption peak. In the absence of disorder, the peak would be very sharp, because (unlike unbound electron-hole pairs) conservation of momentum implies that the oscillator strength for creation of the exciton vanishes unless the centre of mass of the exciton is stationary (see Ref. [16]; this can also be verified using the model discussed in Section 3). The width of the absorption peak in Fig. 1(a) is determined by inhomogeneous broadening due to disorder. The energy of an exciton in the ground state depends upon the width of the potential well in which it is confined, and the well width varies randomly with position in the plane. We assume that the length scale over which the well width varies is large compared with the width of the wells: this implies that the ground state energy of a static exciton at position 


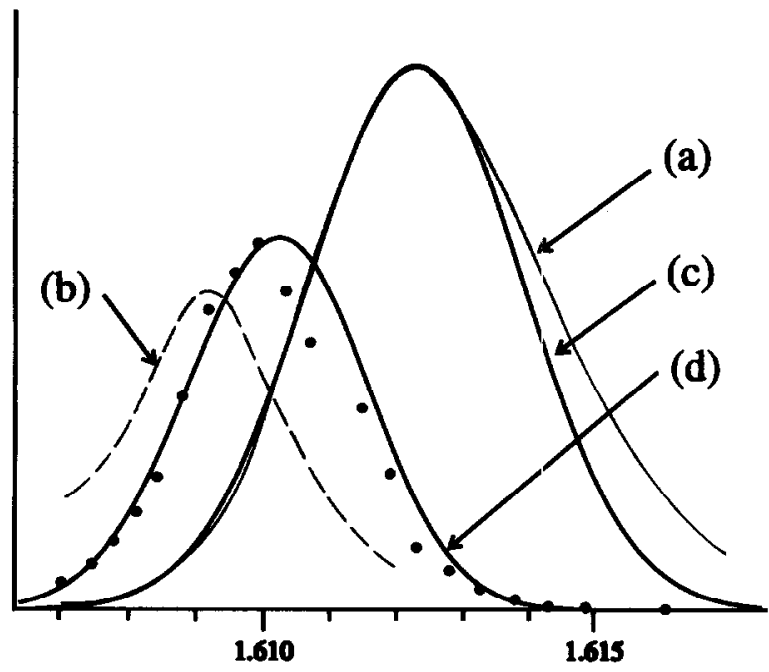

$E / \mathrm{eV}$

Fig. 1. Experimental data from Ref. [15]: (a) absorption spectrum, (b) luminescence spectrum, isolated points-resonant Rayleigh scattering. Theoretical curves: (c) Gaussian fit to absorption spectrum, (d) corresponding distribution of heights of local minima.

$(x, y)$ is a well defined smooth function, which we denote by $E(x, y)$. It is reasonable to assume that the fluctuations of $E(x, y)$ represent contributions from many independent events, and the central limit concept then indicates that the fluctuations are Gaussian. Because the excitons are created with zero centre of mass motion, the absorption spectrum is proportional to the distribution of $E(x, y)$. The curve (c) in Fig. 1 is a fit of a Gaussian curve to the absorption spectrum (a): it fits quite closely.

Now consider the luminescence peak. This is shifted toward lower energies because the excitons can lose energy before they decay. Time-resolved studies of spectral hole burning indicate that the energies of excitons can change over a timescale of typically tens of picoseconds, much shorter than the half-life for decay of excitons, typically several hundred picoseconds $[5,6]$. The predominant mechanism of energy loss for the excitons appears to be by the excitation of phonons: if the absorption spectrum is probed with narrow spectral lines, it is possible to observe features in the luminescence spectrum which are shifted from the probe frequency by multiples of the frequency of the optical phonons [6]. These results justify the following picture of the luminescence process: after the exciton is created at position $\left(x_{0}, y_{0}\right)$ with energy $E_{0}=E\left(x_{0}, y_{0}\right)$, it will move into regions where the potential energy $E(x, y)$ is less than $E_{0}$, and the excess energy $E_{0}-E(x, y)$ appears as kinetic energy. The moving exciton is able to excite phonons and, as it does so, it loses kinetic energy. Eventually, if it does not decay in the meantime, it will end up trapped in a local minimum of the potential energy $E(x, y)$. Because the exciton lifetime is much longer than the timescale associated with energy transfer to phonons, most of the excitons are trapped close to a local minimum of the potential energy $E(x, y)$ when they decay.

These considerations lead to a model in which both the absorption and luminescence spectra are determined by the statistical topography of a Gauss random function, which we will denote by $f(x, y)$, and which represents the energy function $E(x, y)$ after applying linear scaling transformations to $E, x$ and $y$ such that

$$
\langle f\rangle=0,\left\langle f^{2}\right\rangle=1,\left\langle f_{x}^{2}\right\rangle=\left\langle f_{y}^{2}\right\rangle=1 .
$$

The absorption spectrum is proportional to the Gaussian probability distribution of this 
function, whereas the luminescence spectrum is determined by the distribution of heights of its local minima.

The Gaussian random function $f(x, y)$ is characterised completely by its correlation function $C(\mathbf{r})$, which in the isotropic case is a function of $r=\mathbf{r}$ only. The calculations in Refs $[13,10]$ show that the distribution of stationary points depends on the correlation function only through averages of second derivatives. In the isotropic case, after scaling the function so that equation (2) is satisfied, the distribution of stationary points is characterised [10] by a single parameter $a$, where

$$
\left\langle f_{x x}^{2}\right\rangle=\left\langle f_{y y}^{2}\right\rangle=3\left\langle f_{x y}^{2}\right\rangle=3 a .
$$

The distribution of heights of local minima was determined analytically in Refs $[9,10]$ : the result is that

$$
\begin{aligned}
& P_{\min }(f)=\frac{\sqrt{3}}{2 \pi a \sqrt{2 a-1}}\left[-(2 a-1) f \exp \left(\frac{-a f^{2}}{2 a-1}\right)+\frac{a \sqrt{2 \pi a(2 a-1)}}{\sqrt{3 a-1}} \exp \left(\frac{-3 a f^{2}}{2(3 a-1)}\right)\right. \\
& \left.\quad \times \operatorname{erfc}\left(\frac{\sqrt{a}}{\sqrt{2(2 a-1)(3 a-1)}} f\right)+\frac{1}{2} \sqrt{2 \pi(2 a-1)}\left(f^{2}-1\right) \exp \left(-\frac{f^{2}}{2}\right) \operatorname{erfc}\left(\frac{f}{\sqrt{2(2 a-1)}}\right)\right]
\end{aligned}
$$

where $\operatorname{erfc}(x)$ is the complementary error function [17].

The prediction in equation (4) contains the undetermined parameter $a$. In Refs $[9,10]$, it was argued that the annealing process involved in the growth of the heterostructures causes the fluctuations of $E(x, y)$ to be suppressed by a diffusive process: we write

$$
\begin{aligned}
& E(x, y)=\int \mathrm{d} x^{\prime} \int \mathrm{d} y^{\prime} P\left(x-x^{\prime}, y-y^{\prime}\right) E^{\prime}\left(x^{\prime}, y^{\prime}\right), \\
& P(X, Y)=\frac{1}{8 \pi D t} \exp \left(-\frac{X^{2}+Y^{2}}{4 D t}\right)
\end{aligned}
$$

where $E^{\prime}(x, y)$ characterises an initial distribution of well width fluctuations with a much shorter correlation length, $D$ is the diffusion constant and $t$ the annealing time. We may therefore model $E(x, y)$ as the convolution of a white noise function with a Gaussian. It follows that the correlation function of $E(x, y)$ is also a Gaussian, implying that $a=1$.

The distribution of minima in equation (4) with $a=1$ is shown as curve (d) in Fig. 1; the mean and variance have been scaled to correspond to the mean and variance of the Gaussian distribution (c). The curve is not a particularly good fit to the luminescence spectrum (b). The experimental data shown in Fig. 1 are typical: it is usually found that the Stokes shift is somewhat higher than our theoretical prediction [9]. We will make two points about this discrepancy.

First, we propose a qualitative explanation of this observation: the excitons can move into deeper local minima than the ones in which they were initially trapped before they recombine, either by thermally assisted hopping or quantum mechanical tunnelling. This explanation is supported by the literature on time resolved luminescence studies, which show that the Stokes shift initially assumes a value close to our prediction, and then slowly increases [18].

Our second, more important, point concerns resonant Rayleigh scattering experiments on excitons, such as that discussed in Ref. [15]. From the discussion above, it is apparent that it would be desirable to have a more direct probe of the density of local minima of the effective potential to facilitate comparison between theory and experiment. We will now argue that resonant Rayleigh scattering spectra measure the density of local minima of the effective potential. The intensity of Rayleigh scattering from an exciton is proportional to 
the time over which the exciton survives at the energy of the incident radiation. The lifetime of an exciton trapped in a minimum of the effective potential is equal to the lifetime for luminescent decay, typically less than a nanosecond. An exciton which is not trapped may lose energy by exciting phonons, and the lifetime for these processes is much shorter, typically a few tens of picoseconds. The spectra for resonant Rayleigh scattering are proportional to a density of states weighted by the exciton survival time. Because the survival time is very much larger for trapped excitons, the Rayleigh scattering spectrum is weighted very heavily by the density of states for trapped excitons.

The isolated points plotted on Fig. 1 are resonant Rayleigh scattering amplitudes measured at a discrete set of frequencies, taken from data published in Ref. [15]. Both the mean and the variance of these data are remarkably close to the theoretical distribution of local minima, curve (d), which is a plot of equation (4) with $a=1$, and with the mean and variance chosen to match those of the Gaussian fit to the absorption spectrum.

\section{MODEL FOR EXCITON LUMINESCENCE MATRIX ELEMENTS}

We consider a solid consisting of $N$ atoms, each of which can exist in four different states:

1. Neutral and unexcited, $|0\rangle$.

2. With additional electron (in the conduction band), $|-\rangle$.

3. With an electron removed (i.e. with hole in valence band), $|+\rangle$.

4. Excited atom (or Frenkel exciton), with an electron in the conduction band and a hole in the valence band, $\left|e_{\Gamma}\right\rangle=| \pm\rangle$.

We consider a Hilbert space for the solid with $4^{N}$ basis vectors, consisting of all possible combinations of these four states of the $N$ individual atoms: for example, a state with two electrons, a hole and an exciton could be written as $|0,0,-, 0,0, \ldots, 0,0,+, 0, \pm, 0,-, 0, \ldots\rangle=$ $\left|n_{1}, n_{2}, n_{3} ; n_{3}, n_{4}\right\rangle$, where $n_{1}$ and $n_{2}$ are the positions of the electrons, $n_{4}$ is the position of the hole and $n_{3}$ is the position of the Frenkel exciton.

This model is reasonable for insulators in which the electrons are tightly bound to individual atoms. This description can also be carricd over directly to semiconductors, if the electron and hole states localised on individual atoms are replaced by Wannier states derived by integrating over the conduction and valence band wavefunctions, respectively [16].

In a semiconductor, the excitons are typically of the Wannier type, in which the electrons and holes, although correlated, are not bound to the same atomic orbital. The Wannier exciton state $\left|e_{W}\right\rangle$ is a superposition of electron and hole states of the form

$$
\left|e_{W}\right\rangle=\sum_{n_{1}, n_{2}} c_{n_{1}, n_{2}}\left|n_{1} ; n_{2}\right\rangle
$$

where $n_{1}$ and $n_{2}$ are the positions of the electron and hole, respectively. In the case of the weakly localised Wannier exciton, the coefficients $c_{n_{1}, n_{2}}$ can be approximated by a continuous wavefunction $\psi(\mathbf{x}, \mathbf{y})$, where $\mathbf{x}$ and $\mathbf{y}$ are the locations of atoms with labels $n_{1}$ and $n_{2}$. The wavefunction $\psi(\mathbf{x}, \mathbf{y})$ is an eigenfunction of an effective Hamiltonian [16]

$$
\hat{H}=\frac{1}{2 m_{\mathrm{e}}} \mathbf{p}_{\mathrm{e}}^{2}+\frac{1}{2 m_{\mathrm{h}}} \mathbf{p}_{\mathrm{h}}^{2}+\frac{\mathrm{e}^{2}}{4 \pi \epsilon_{0}} \frac{1}{\left|\mathbf{r}_{\mathrm{e}}-\mathbf{r}_{\mathrm{h}}\right|}+V_{\mathrm{e}}\left(\mathbf{r}_{\mathrm{e}}\right)+V_{\mathrm{h}}\left(\mathbf{r}_{\mathrm{h}}\right) .
$$

The intensity of emission from an exciton state is proportional to the square of the dipole matrix element $\left\langle e_{\mathrm{w}}|\hat{X}| 0\right\rangle$ for the transition between the exciton state and the ground state of the system, summed over three orthogonal choices for the coordinate $X$. This matrix 
element can be expressed in terms of the dipole matrix elements of the localised basis states. We assume that the dipole matrix element for the basis states is

$$
\left\langle 0|\hat{X}| n_{1} ; n_{2}\right\rangle=\epsilon \delta_{n_{1} n_{2}},
$$

i.e. the dipole matrix element for recombination is assumed to be negligible, unless the electron and hole are on the same atomic site. Most crystals have centres of symmetry, and the dipole matrix element $\langle 0|\hat{X}| n ; n\rangle$ vanishes if the atomic orbitals or Wannicr functions defining the valence and conduction bands have the same parity with respect to the $X$ coordinate. The atomic orbitals associated with successive bands typically have opposite parity with respect to one of the coordinates, so that for at least one choice of the coordinate $X$ the matrix element considered in equation (8) does not vanish because of symmetry considerations.

The required matrix element can now be calculated using equation (8):

$$
\left\langle 0|\hat{X}| e_{\mathrm{w}}\right\rangle=\sum_{n_{1} n_{2}} c_{n_{1} n_{2}}\left\langle 0|\hat{X}| n_{1} ; n_{2}\right\rangle=\epsilon \sum_{n} c_{n n} \sim \epsilon^{\prime} \int \mathrm{d} \mathbf{r} \psi(\mathbf{r}, \mathbf{r}),
$$

where $\epsilon^{\prime}$ is another constant. The matrix element is therefore proportional to the amplitude for the electron and hole to be at the same site.

We now consider the case of excitons in heterostructures, where the excitons are trapped in a layer of low bandgap material between regions of higher bandgap. Imperfections of the growth process result in random fluctuations of the layer width; we will assume that the fluctuations of the layer width are on a larger scale than the size of the exciton [10]. We therefore use the model

$$
\begin{aligned}
V_{\mathrm{e}}\left(\mathbf{r}_{\mathrm{e}}\right)+V_{\mathrm{h}}\left(\mathbf{r}_{\mathrm{h}}\right) & =v_{\mathrm{e}}\left(z_{\mathrm{e}}\right)+v_{\mathrm{h}}\left(z_{\mathrm{h}}\right)+u_{\mathrm{e}}\left(\mathbf{x}_{\mathrm{e}}\right)+u_{\mathrm{h}}\left(\mathbf{x}_{\mathrm{h}}\right) \\
& \sim v_{\mathrm{e}}\left(z_{\mathrm{e}}\right)+v_{\mathrm{h}}\left(z_{\mathrm{h}}\right)+u_{\mathrm{e}}(\mathbf{X})+u_{\mathrm{h}}(\mathbf{X}),
\end{aligned}
$$

where $\mathbf{x}_{\mathrm{e} / \mathrm{h}}=\left(x_{\mathrm{e} / \mathrm{h}}, y_{\mathrm{e} / \mathrm{h}}\right)$ are the electron/hole coordinates in the $(x, y)$ plane, and $\mathbf{X}$ and $\mathbf{x}$ are the corresponding centre of mass and relative coordinates. In the second line, the assumption that the variation of the potentials $u_{\mathrm{e} / \mathrm{h}}$ is slow on the scale of the exciton diameter justifies the approximation $\mathbf{x}_{\mathbf{e}} \sim \mathbf{x}_{\mathbf{h}} \sim \mathbf{X}$. The effective Hamiltonian can then be written in the separated form

where

$$
H\left(\mathbf{r}_{\mathbf{e}}, \mathbf{r}_{\mathbf{h}}, \mathbf{p}_{\mathbf{e}}, \mathbf{p}_{\mathbf{h}}\right)=H_{0}+H_{1}
$$

$$
H_{0}=\frac{1}{2 \mu} \mathbf{p}^{2}+\frac{\mathrm{e}^{2}}{4 \pi \epsilon_{0}|\mathbf{r}|}+v_{\mathrm{e}}\left(z_{\mathrm{e}}\right)+v_{\mathrm{h}}\left(z_{\mathrm{h}}\right), \quad H_{1}=\frac{1}{2 M} \mathbf{P}^{2}+V_{\mathrm{eff}}(\mathbf{X}),
$$

and $V_{\text {efr }}(X, Y)=u_{\mathrm{e}}(X, Y)+u_{\mathrm{h}}(X, Y), \mathbf{P}$ is the momentum conjugate to $\mathbf{X}=(X, Y)$, and $\mu$ and $M$ are the reduced and total masses. The corresponding solution of the Schrödinger equation is a product of an exciton wavefunction $\chi\left(x, y, z_{\mathrm{e}}, z_{\mathrm{h}}\right)$, and a wavefunction $\phi(X, Y)$ for the centre of mass motion in the $(X, Y)$ plane. Equations (9) and (11) show that the transition strength for exciton recombination is of the form

$$
I \sim\left|\left\langle 0|\hat{X}| e_{\mathrm{w}}\right\rangle\right|^{2}=C\left|\int \mathrm{d} X \int \mathrm{d} Y \phi(X, Y)\right|^{2},
$$

where the constant $C$ is the same for all exciton states.

We first discuss the interpretation of this result for a non-disordered system. In this case, the centre of mass wavefunctions are $\phi(\mathbf{X})=A^{-1 / 2} \exp [\mathbf{i k . X}]$, where $A$ is the area of the sample. The recombination transitions only occur from the ground state, for which 
$\phi(X, Y)=A^{-1 / 2}$, because the integral in equation (12) vanishes for all of the other states. Equation (12) then implies that the transition rate for recombination from the centre of mass ground state is proportional to the area of the system, whereas the rate for all of the other possible states is zero. This is in accord with the expectation that the sum of the transition rates for a system of area $A$ should be proportional to $A$.

Now consider the case of a disordered system. We will use the same model as in Refs [9] and [10]: we assume that the excitons interact strongly with phonons, and that mobile excitons rapidly lose energy by exciting phonons. Most of the exciton recombination therefore occurs after the excitons have become trapped in minima of the effective potential $V_{\text {eff }}(X, Y)$. We will assume that the effective potential for the centre of mass motion is an isotropic Gauss random function. The form of the effective potential in the neighbourhood of its minima can be approximated by a quadratic form: if the origin of the $(X, Y)$ plane is shifted to lie at the minimum, we write

$$
V_{\text {eff }}(X, Y) \sim V_{0}+\frac{1}{2}\left[V_{X X} X^{2}+V_{Y Y} Y^{2}+2 V_{X Y} X Y\right]=V_{0}+\frac{1}{2} \mathbf{X}^{\mathrm{T}} \tilde{M} \mathbf{X},
$$

where $\tilde{M}$ is the Hessian matrix of second derivatives evaluated at the minimum. The exciton recombination occurs from a ground state of the centre of mass motion trapped in this minimum, for which the wavefunction $\phi(X, Y)$ is a harmonic oscillator ground state, which satisfies

$$
\int \mathrm{d} X \int \mathrm{d} Y \phi(X, Y)=c[\operatorname{det} \tilde{M}]^{-1 / 8},
$$

where $c$ is independent of the local environment in which the exciton is trapped. The distribution of transition strengths $I$ for trapped excitons is therefore determined from the distribution of determinants of the Hessian matrix at minima:

$$
I \sim|D|^{-1 / 4}, \quad D=\operatorname{det} \tilde{M} .
$$

\section{DISTRIBUTIONS OF INTENSITIES}

\subsection{Distribution of quadratic forms at stationary points:}

Methods for calculating properties of point singularities such as minima are well known: the one-dimensional and two-dimensional cases are discussed by Rice [12] and LonguetHiggins [13]. We will calculate the distribution of the trace $T$ and determinant $D$ of the Hessian matrix

$$
\tilde{M}=\left(\begin{array}{ll}
f_{x x} & f_{x y} \\
f_{x y} & f_{y y}
\end{array}\right)
$$

describing the second derivatives $f_{x x}, f_{x y}, f_{y y}$ of an isotropic Gauss random function $f(x, y)$ at its stationary points.

We follow the approach and notation of Ref. [10]: the distribution of extrema is determined by the joint probability distribution $P\left(f, f_{x}, f_{y}, f_{x x}, f_{y y}, f_{x y}\right)$ of the function $f$, its first derivatives $f_{x}$ and $f_{y}$, and its second derivatives evaluated at the same point $(x, y)$. By a simple adaptation of the calculation in Ref. [10], the joint distibution of the trace and determinant is

$$
P(T, D)=\frac{1}{\mathcal{N}} \int \mathrm{d} f \int \mathrm{d} f_{x x} \int \mathrm{d} f_{y y} \int \mathrm{d} f_{y y} P\left(f, 0,0, f_{x x}, f_{y y}, f_{x y}\right)|d| \delta(D-d) \delta(T-t),
$$

where $\mathcal{N}$ is a normalisation factor, $t=\left(f_{x x}+f_{y y}\right)$ and $d=f_{x x} f_{y y}-f_{x y}^{2}$. The function is assumed to be scaled so that equation (2) is satisfied. 
In terms of the variables $R=t / 2, X=\left(f_{x x}-f_{y y}\right) / 2$ and $Y=f_{x y}$, the probability distribution in equation (17) was obtained in Ref. [10] as

$$
P\left(f, 0,0, f_{x x}, f_{y y}, f_{x y}\right)=\frac{1}{(2 \pi)^{3} a \sqrt{2 a-1}} \exp \left(-\frac{f^{2}}{2}\right) \exp \left(-\frac{(f+R)^{2}}{2(2 a-1)}\right) \exp \left(-\frac{X^{2}+Y^{2}}{2 a}\right) .
$$

The integral in equation (17) can now be evaluated easily. The normalised probability density is

$$
P(T, D)=\frac{1}{32 a^{2}} \sqrt{\frac{3}{\pi a}}|D| \exp \left(\frac{D}{2 a}\right) \exp \left(-\frac{3 T^{2}}{16 a}\right) \Theta\left(D-\frac{T^{2}}{4}\right),
$$

where $\Theta(x)$ is a step function, decreasing from 1 to 0 at $x=0$.

\subsection{Distribution of oscillator strengths}

Now we will use the results of Section 3 to calculate the distribution of transition strengths for excitons. The distribution of the determinant $D$ of the Hessian matrix at stationary points is obtained by integrating equation (19) over $T$ :

$$
P(D)=\int_{-\infty}^{\infty} \mathrm{d} T P(T, D)=\frac{1}{8 a^{2}}|D| \exp \left(\frac{D}{2 a}\right)\left[\Theta(D)+\Theta(-D) \operatorname{erfc}\left(\frac{1}{2} \sqrt{3 D / a}\right)\right] .
$$

It is noteworthy that the parameter $a$ only appears in the ratio $D / a$ in this probability measure: it therefore sets the scale of the distribution of $D$ but does not alter its functional form. The distribution of intensities is therefore insensitive to this parameter, and we may take $a=1$.

The minima are those extrema for which $D>0$ and $T>0$. The distribution of intensitics $I \sim|D|^{-1 / 4}$ follows immediately from this expression, considering only the branch with $D>0$. The result, with arbitrary scale for the intensities, is given by equation (1). Numerically, the mean value of the intensities with distribution given by equation (1) is found to be $\langle I\rangle=0.699 \ldots$. The distribution of equation (1) is plotted in Fig. 2(a).

\subsection{Relation to sea glitter}

The analysis of Gauss random functions in two dimensions was largely motivated by the desire to achieve a statistical understanding of the surface of the sea. It is natural to ask which experimentally observable property of the sea surface corresponds most closely to our
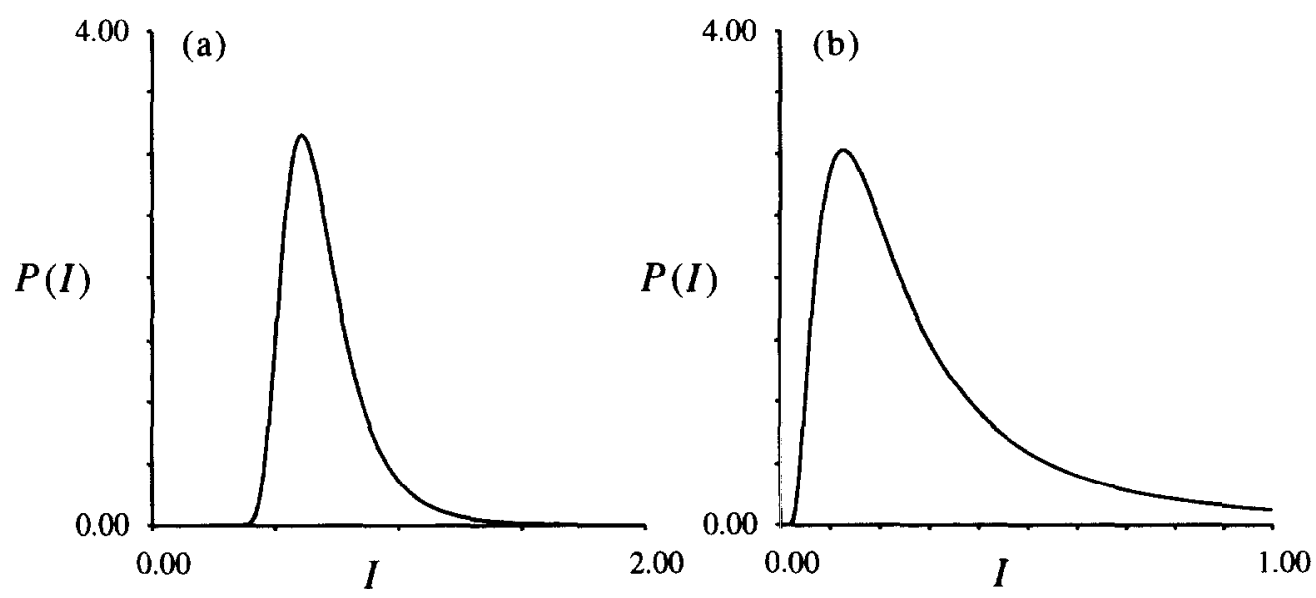

Fig. 2. Predicted distribution of intensities of (a) exciton luminescence, (b) sea glitter sparkles. 
distribution of exciton transition intensities and to compare the two results. The corresponding property is 'sea glitter' [14], which is the pattern of reflections of the sun on the ocean surface seen from a high flying aircraft.

To simplify the discussion, we will assume that the sun is overhead and that the sea glitter is observed by looking vertically downwards. If the radius of the sun subtends an angle $\epsilon$, the observer sees a bright area on the sea suface due to reflected sunlight, whenever the angle of the sea surface is smaller than $\epsilon / 2$. There is therefore a bright spot on the sea surface in the neighbourhood of every maximum, minimum or saddle point: each of these bright spots is seen as an ellipse, of area $A \sim|\operatorname{det} \tilde{M}|^{-1}$, where $\tilde{M}$ is the Hessian matrix at the stationary point. The quantity which corresponds to the distribution of exciton intensities is the distribution of integrated intensities $I \sim A$ of the reflected images of the sun. The distribution of determinants, for all types of stationary points, is given by equation (20) and the corresponding distribution of $I \sim|D|^{-1}$ is

$$
P(I)=\frac{1}{8} I^{-3}\left[\exp \left(-\frac{1}{2 I}\right)+\exp \left(\frac{1}{2 I}\right) \operatorname{erfc}\left(\frac{1}{2} \sqrt{3 / I}\right)\right]
$$

Note that, once again, the distribution is independent of the parameter $a$ : this result is therefore universal for isotropic sea surfaces. This distribution is plotted in Fig. 2(b). Again, the normalisation of the intensity distribution is arbitrary and, in this case, we find numerically that $\langle I\rangle=0.433 \ldots$. This result is very different from the distribution of exciton intensities. The reasons for the difference are that all of the extrema contribute and that there is a different relationship between $I$ and $D=\operatorname{det} \tilde{M}$.

\section{REFERENCES}

1. Dingle, R., Festkörperprobleme, 1975, 15, 21.

2. Göbel, E. O., Optical properties of excitons in quantum wells. In Excitons in Confined Systems. Springer, Heidelberg, 1988.

3. Weisbuch, C., Dingle, R., Gossard, A. C. and Weigmann, W., Solid State Commun., 1981, 38, 709.

4. Singh, J., Bajaj, K. K. and Chadhuri, S., Appl. Phys. Lett., 1984, 44, 805.

5. Hegarty, J. and Sturge, M. D., J. Opt. Soc. Amer. B, 1985, 2, 1143-1154.

6. O'Donnell, K. P. and Henderson, B., J. Luminescence, 1992, 52, 133-146.

7. Gammon, D., Snow, E. S. and Katzer, D. S., Appl. Phys. Lett., 1995, 67, 2391-2393.

8. Gammon, D., Snow, E. S., Shanabrook, B. V., Katzer, D. S. and Park, D., Phys. Rev. Lett. 1996. 76, 3005-3008.

9. Fang Yang, M., Wilkinson, E., Austin, J. and O'Donnell, K. P., Fhys. Rev. Lett., 1993, 70, 323-326.

10. Wilkinson, M., Fang Yang, E., Austin, J. and O'Donnell, K. P., J. Phys.: Condensed Matter, $1992,4,8863-8878$.

11. Porter, C. E. (ed.), Statistical Theories of Spectra: Fluctuations. Academic Press, New York, 1965.

12. Rice, S. O., Bell. Sys. Tech. J., 1945, 24, 46-156.

13. Longuet-Higgins, M. S., Philos. Trans. Roy. Soc. London Ser. A, 1957, 249, 321-387.

14. Cox, C. and Munk, W., J. Opt. Soc. Am., 1954, 44, 838.

15. Hegarty, J., Sturge, M. D., Weisbuch, C., Gossard, A. C. and Weigmann, W., Phys. Rev. Lett., 1982, 49, 930-932.

16. Knox, R. S., Theory of Excitons. Academic Press, New York, 1963.

17. Abramowitz, M. and Stegun, I. A., Handbook of Mathematical Finctions. Dover, New York, 1970.

18. Stanley, R. P., Hegarty, J., Fischer, R., Feldmann, J., Göbel, E. O., Feldmann, R. D. and Austin, R. F., Phys. Rev. Lett., 1991, 67, 128. 